\title{
Modulation Classification of Satellite Communication Signals Using Cumulants and Neural Networks
}

\author{
Aaron Smith, Michael Evans, and Joseph Downey \\ Information and Signal Processing Branch \\ NASA Glenn Research Center \\ Cleveland, $\mathrm{OH} 44135$ \\ \{aaron.smith, michael.a.evans-1, and joseph.a.downey\}@nasa.gov
}

\begin{abstract}
National Aeronautics and Space Administration (NASA) is investigating cognitive technologies for their future communication architecture. These technologies are expected to reduce the operational complexity of the network, increase science data return, and reduce interference to self and others. In order to increase situational awareness, signal classification algorithms could be applied to identify users and distinguish sources of interference. As a preliminary step, we seek to develop a system with the ability to discern signals typically encountered in satellite communication. Proposed is an automatic modulation classifier which utilizes higher order statistics (cumulants) and an estimate of the signal-to-noise ratio. These features are extracted from baseband symbols and then processed by a neural network for classification. The modulation types considered are phaseshift keying (PSK), amplitude and phase-shift keying (APSK), and quadrature amplitude modulation (QAM). Physical layer properties specific to the Digital Video Broadcasting - Satellite - Second Generation (DVB-S2) standard, such as pilots and variable ring ratios, are also considered. This paper will provide simulation results of a candidate modulation classifier, and performance will be evaluated over a range of signal-to-noise ratios, frequency offsets, and nonlinear amplifier distortions.
\end{abstract}

\section{INTRODUCTION}

A current effort at National Aeronautics and Space Administration (NASA) is the development of cognitive communication systems. These systems use knowledge of the signal environment, temporal and spatial channel parameters, and user needs to optimize communication links dynamically. In current systems, transmitter and receiver parameters are generally static in nature and become sub-optimal as the needs of the users and the environment change with time. Automatic signal detection and classification will assist a cognitive system by enabling technologies such as automatic receiver configuration, interference mitigation, and spectrum management.

Though modulation classification is a well-studied field [1], there remain many unsolved problems in reaching a solution that is both generalizable and computationally practical. In this preliminary investigation, we focus on phase-shift keying (PSK), amplitude and phase-shift keying (APSK), and quadrature amplitude modulation (QAM), as these modulation types are commonly found in space communication systems. We assume that our signal can be isolated, that the user is able to recover symbol timing, and that a signal-to-noise ratio estimate is available prior to classification.

A primary consideration in a classification scheme is the feature set extracted from a signal. Common features include spectral statistics [2], non-cyclic cumulants [3]-[5], cycliccumulants [6]-[9], Cyclic Auto-Correlation (CAC) function and Spectral Coherence Function (SCF) [10] [11], and Fourierwavelet transforms [12]. For this study, we chose to use cumulant-based features, as they have been shown effective at classifying modulation order.

In recent years, the Digital Video Broadcasting - Satellite Second Generation (DVB-S2) standard [13] has seen increased interest at NASA [14]. This standard uses the APSK modulation scheme. Literature on the classification of modulation sets that include APSK is sparse, however in [5] the authors describe a similar effort using a decision tree classifier based on cumulants. That work follows the methods described in [3]. Our investigation utilizes a neural network to automate the thresholding process. We also include APSK signals using the ring ratios defined in the DVB-S2 standard. Furthermore, we seek to extend the work done in [5] by investigating the impact of DVB-S2 headers and pilots on cumulant values, determining classifier resilience to $E_{s} / N_{0}$ estimation error, and evaluating performance when the signal has experienced a nonlinear amplifier distortion.

In Section II we present our signal model. In this work, we assume the user was able to recover carrier frequency and symbol timing. In future work, we expect to recover these parameters using properties of the conjugate and nonconjugate versions of the $\mathrm{CAC}$, similar to the preprocessing steps taken in [10]. Though the authors in [10] did not recover symbols, common methods could use symbol rate estimates to identify symbol timing.

Section III discusses the features extracted from received signals. In this section, we briefly demonstrate a method for generating arbitrary mixed cumulants and display the 
theoretical cumulants for our modulation set. Following this, we describe the structure of our classifying neural network.

In the DVB-S2 standard, the APSK modulation is defined for a set of ring ratios. In our early results, we found that a neural network trained on a specific ring ratio would fail to classify APSK signals which used a different ring ratio. This seems an obvious outcome, as the ring ratios used impact the theoretical cumulant values of the signal. To resolve this, we trained the neural networks by randomly selecting from the set of possible ring ratios used in the DVB-S2 standard. In the simulations that follow, all neural networks were trained in this manner.

The DVB-S2 physical layer breaks the stationary assumption of the received signal due to the inclusion of header and pilot sequences. In Section IV-F, we analyze the effect of the DVB-S2 physical layer on cumulant estimates. In that same section, we also attempt to classify the modulation type of DVB-S2 signals with pilots both enabled and disabled.

In [3] and [5], the authors assume knowledge of the signalto-noise ratio and use this to scale the symbol sequence such that it has unit average power. Our method is similar in that we assume an available estimate of $E_{s} / N_{0}$, but instead of scaling the received signal, we simply normalize the received signal plus noise to have unit average power and train the network using $E_{s} / N_{0}$ as a feature. In section IV-D, we investigate the degradation associated with imperfect $E_{s} / N_{0}$ estimation.

During transmission, it is common to operate an amplifier in the nonlinear region to maximize transmit power. For modulations with multiple amplitude levels like APSK and QAM, this causes amplitude-dependent compression and phase rotation. This study simulates this by applying a Saleh model [15] using coefficients from an operational Traveling Wave Tube Amplifier (TWTA) [16]. In Section IV-E, we present the classification degradation caused by this nonlinear amplification.

\section{SignAl MODEL}

In the classification simulations that follow, we assume that a signal was detected, isolated, and processed such that we are able to recover complex symbols. We leave the possibility of a residual carrier frequency offset and fixed phase offset. After this assumed preprocessing, we model the symbols with noise as

$$
r[n]=A e^{j\left(2 \pi f_{o} n T+\phi\right)} x[n]+g[n]
$$

where $A$ is an unknown amplitude scalar, $f_{o}$ is a residual carrier frequency, $T$ is the symbol period, $\phi$ is our constant phase offset, and $g[n]$ is a complex-valued additive white Gaussian noise process. We then normalize to unit average power such that

$$
y[n]=\frac{r[n]}{\sqrt{\frac{1}{N} \sum_{n=0}^{N-1}|r[n]|^{2}}}
$$

We assume a modulation set

$$
\begin{array}{r}
\Omega=\{16-A P S K, 32-A P S K, \\
2-P S K, 4-P S K, 8-P S K, \\
16-Q A M, 64-Q A M\}
\end{array}
$$

The sequence $\{x[n]\}_{n=0}^{N-1}$ contains $N$ equiprobable, complex, zero-mean symbols which exist in the finite-alphabet of a modulation scheme in the set $\Omega$.

The presence of noise in the received signal provides the motivation to capture longer sequences during classification. However, our ability to classify an incoming signal degrades as the accumulated phase error $\left(2 \pi f_{o} N T\right)$ increases and provides a counterargument to longer signal captures. If an appropriate frequency estimate is not available, a composite signal

$$
z[n]=y^{*}[n-1] y[n]
$$

is proposed in [17], where * denotes the complex conjugate. This will turn a constant frequency offset into a fixed phase offset, though a time-varying $f_{o}$ will still cause $z[n]$ to rotate in the IQ plane during capture. As noted in [3], we will need more data when using the sequence $\{z[n]\}$ (compared with $\{y[n]\}$ ), as this is equivalent to computing a higher order cumulant, or the cumulant of a correlated sequence.

\section{ClassificAtion}

\section{A. Features}

A stationary random process can be characterized by its moments and/or cumulants. We seek to leverage this in order to reduce the dimensionality of our classification problem. The mixed moments of a complex-valued, stationary random process $s[n]$ are defined as

$$
M_{p q}=E\left[s[n]^{(p-q)}\left(s^{*}[n]\right)^{q}\right]
$$

which is approximated by

$$
\hat{M}_{p q}=\frac{1}{N} \sum_{n=0}^{N-1} s[n]^{(p-q)}\left(s^{*}[n]\right)^{q}
$$

We generate arbitrary mixed cumulants by defining a set of random variables which consist of either $s[n]$ or $s^{*}[n]$.

$$
S=\{\underbrace{s[n], \ldots, s[n]}_{\mathrm{p}-\mathrm{q} \text { terms }}, \underbrace{s^{*}[n], \ldots, s^{*}[n]}_{\mathrm{q} \text { terms }}\}
$$

Then, the cumulant is generated via the moment-to-cumulant function

$$
C_{p q}(S)=\sum_{\pi}(-1)^{|\pi|-1}(|\pi|-1) ! \prod_{B \in \pi} E\left[\prod_{i \in B} S_{i}\right]
$$

where $\pi$ steps through all partitions of $S . B$ is a set within a particular partition $\pi$ and the number of sets in $\pi$ is equal to $|\pi| . E[\cdot]$ is the statistical expectation and $S_{i}$ is the $i^{t h}$ element in $S$. For example, if $S=\left\{s_{1}, s_{2}\right\}$, then the possible partitions 


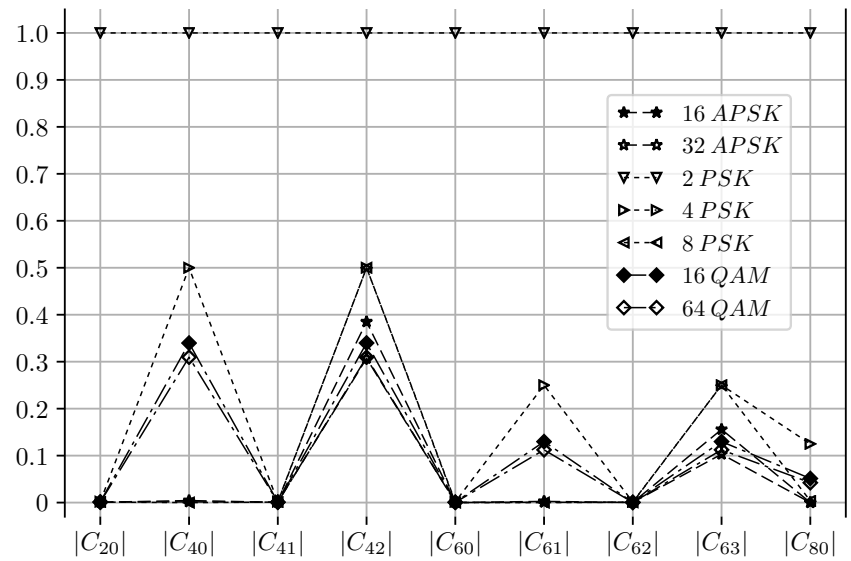

Fig. 1. Theoretical cumulant magnitudes of $y[n]$. Each cumulant has been normalized such that the largest value is equal one.

would be $\left\{\left(s_{1}, s_{2}\right)\right\}$ and $\left\{\left(s_{1}\right),\left(s_{2}\right)\right\}$. The operations in (8) would then be

$$
\begin{array}{r}
C_{p q}\left(s_{1}, s_{2}\right)= \\
(-1)^{1-1}(1-1) ! E\left[s_{1} s_{2}\right]+ \\
(-1)^{2-1}(2-1) ! E\left[s_{1}\right] E\left[s_{2}\right] \\
=E\left[s_{1} s_{2}\right]-2 E\left[s_{1}\right] E\left[s_{2}\right]
\end{array}
$$

If $s 1=s[n]$ and $s 2=s^{*}[n]$, by using our definition in (5) and the assumption of a zero-mean process, we arrive at the expected result of

$$
C_{21}=M_{21}
$$

Assuming zero frequency offset, both $y[n]$ and $z[n]$ are stationary, zero-mean random processes, and we estimate their mixed cumulants in the manner as above. See [9] and [18] for more detailed information on moments and cumulant generation. In Figures 1 and 2, we display the normalized, noiseless, cumulant magnitudes for each modulation in $\Omega$. Our simulations utilize feature vectors which contain the following cumulant magnitudes: $\left|C_{20}\right|,\left|C_{40}\right|,\left|C_{41}\right|,\left|C_{42}\right|,\left|C_{60}\right|,\left|C_{61}\right|,\left|C_{62}\right|,\left|C_{63}\right|,\left|C_{80}\right|$.

In addition to the cumulants listed above, we also require an $E_{s} / N_{0}$ estimate as an element in our feature vector. This is similar to the approach taken in [3], except we allow the neural network to learn cumulants as a function of $E_{s} / N_{0}$ instead of scaling the received signal. In Section IV-D we evaluate the estimation error.

\section{B. Neural Network Classifier}

Our neural network model is a feed-forward, backpropagation multilayer perceptron network. The input is a feature vector of length ten. The next three layers are densely connected with a width of 40 and tanh activation functions. Following this is the output layer of width seven $(|\Omega|)$ and is also densely connected with softmax activation functions. We used a categorical cross-entropy loss function and an Adam optimizer [19]. Classification is performed by indexing

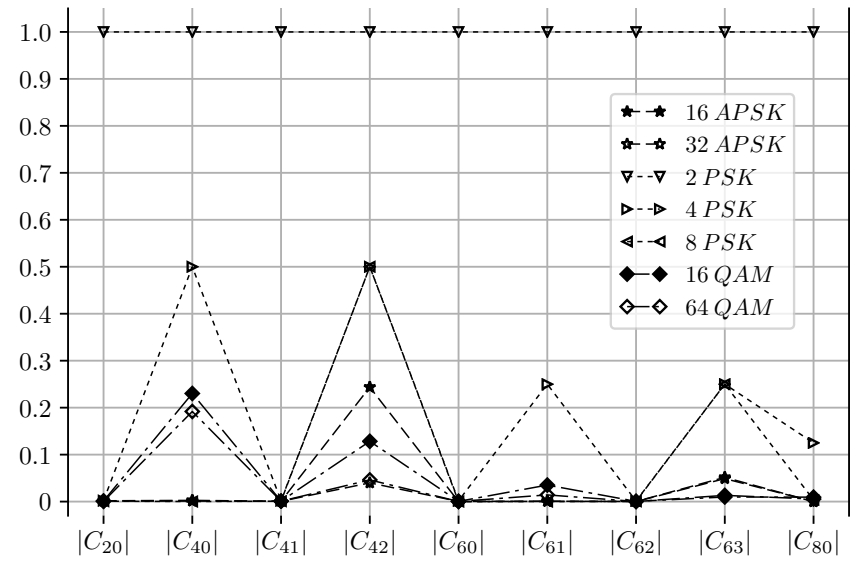

Fig. 2. Theoretical cumulant magnitudes of $z[n]$. Each cumulant has been normalized such that the largest value is equal one.

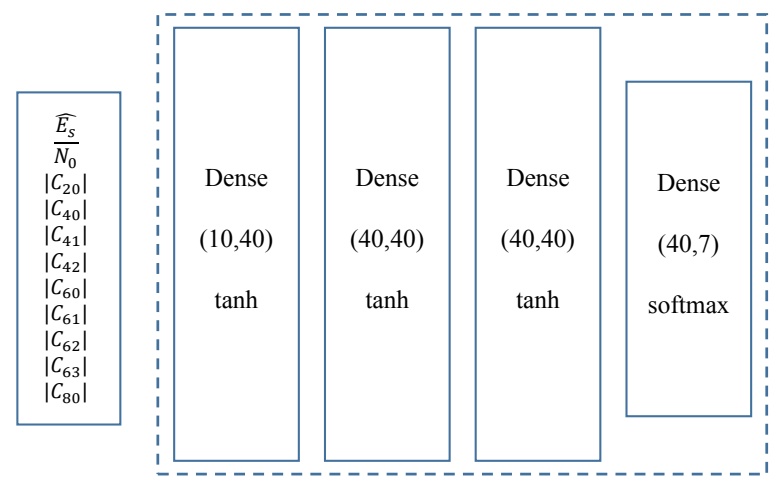

Fig. 3. Classifier architecture. The neural network model takes a ten element feature vector as input and returns a vector of length seven, which is our modulation set size. The modulation is determined by indexing $\Omega$ by the argmax of the output vector.

$\Omega$ with the argmax of the output of the softmax layer. In the simulations presented, we held the model constant. All models have the structure shown in Figure 3.

\section{Simulation Results}

To generate a training set, we chose a capture length $N$ and created 15,000 instances of $\{r[n]\}$ (1) with zero frequency offset and a random constant phase offset. The sequence length used in training was equal to the sequence length of the vectors being classified by a particular classifier. Noise was added such that the $E_{s} / N_{0}$ parameter of the 15,000 $\{r[n]\}$ sequences could be modeled as a uniform continuous random variable in the range $[-3,20] \mathrm{dB}$. The signal $r[n]$ is then converted to $y[n]$ via average power normalization, for each instance. A feature vector $\left(F_{N, y}\right)$ was formed by appending the $E_{s} / N_{0}$ to the cumulant values operated on the sequence $\{y[n]\}_{n=0}^{N-1}$. We then convert $y[n]$ to $z[n]$ as described in (4) and we saved that feature vector $\left(F_{N, z}\right)$ as well. As is discussed in Section IV-B, classifiers based on $z[n]$ required more data for classification. Therefore, we did not create training sets based on $z[n]$ for small values of $N$. Since all the classifiers used 


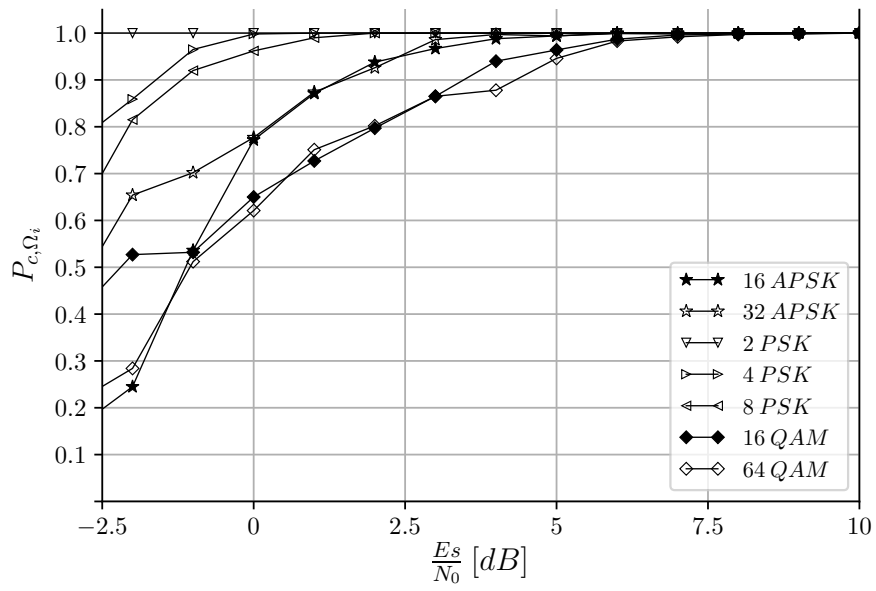

Fig. 4. Modulation specific probability of correct classification, as defined in (11). Cumulants were estimated on the sequence $\{y[n]\}$ with $N=5 k$.

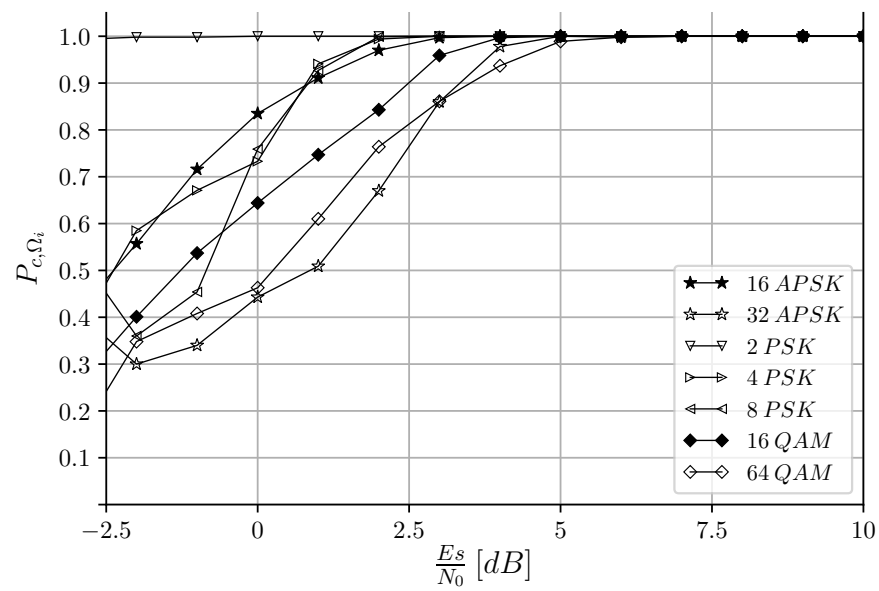

Fig. 5. Modulation specific probability of correct classification, as defined in (11). Cumulants were estimated on the sequence $\{z[n]\}$ with $N=80 k$.

the same neural network model, the training sets can be used to identify the classifier. The training sets/classifiers created were: $F_{1 k, y}, F_{2.5 k, y}, F_{5 k, y}, F_{10 k, y}, F_{20 k, y}, F_{40 k, y}, F_{80 k, y}$, $F_{10 k, z}, F_{20 k, z}, F_{40 k, z}, F_{80 k, z}$.

\section{A. Classification by Modulation}

To evaluate our ability to classify individual modulation types, we generated an evaluation set which consisted of 1,000 instances of $F_{N, y}$ and $F_{N, z}$, for each modulation type, for each $E_{s} / N_{0}$ value in the set $\{-3,-2, \ldots 19,20\} \mathrm{dB}$. Figure 4 and Figure 5 show our results for $F_{5 k, y}$ and $F_{80 k, z}$, respectively. We contrast these sequence lengths in an attempt to compare the outputs when classification ability is roughly equal.

From these graphs, it is clear that 2-PSK is the most easily recognized modulation in our set. We found that the 4-PSK and 8-PSK modulation types were more easily identified (at lower $E_{s} / N_{0}$ values) when utilizing $y[n]$. It is also easily seen

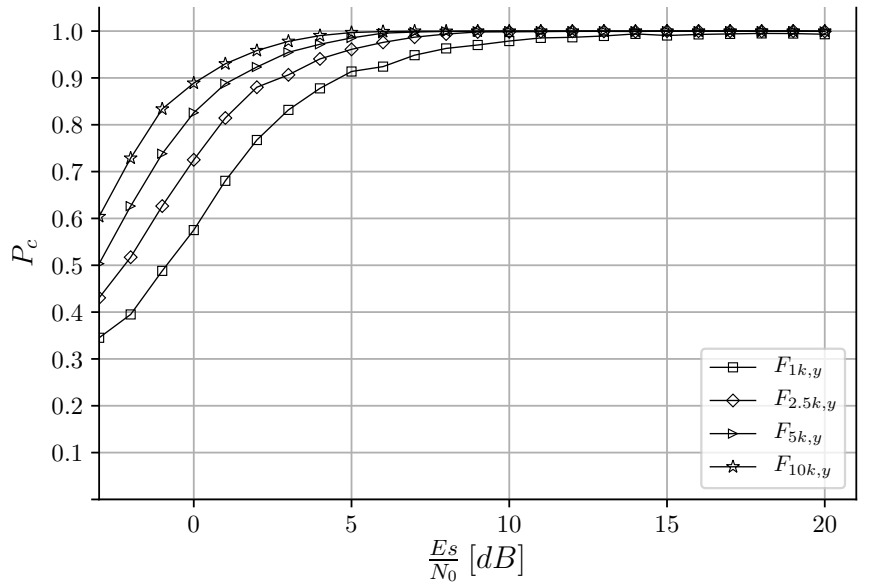

Fig. 6. Probability of correct classification as defined in (12). Cumulants were estimated on the sequence $\{y[n]\}$. The $F_{5 k, y}$ curve is the average of the values found in Figure 4.

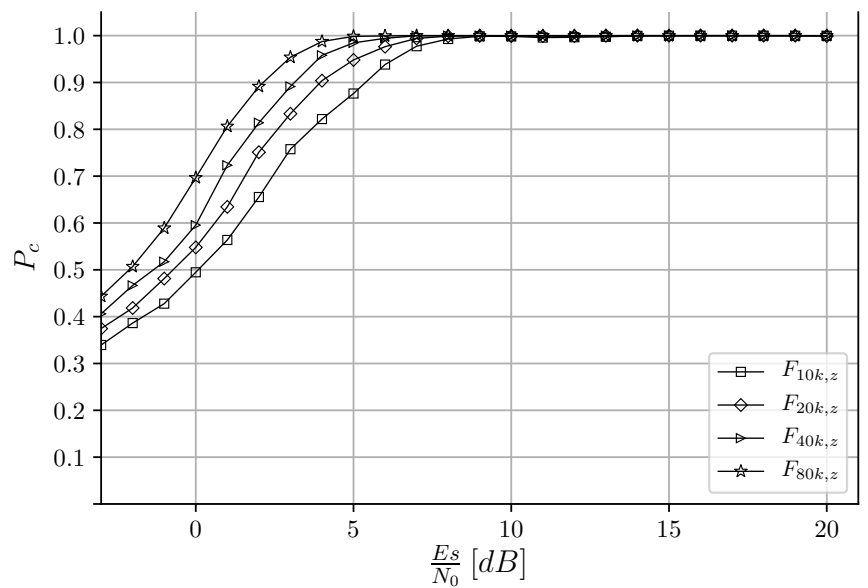

Fig. 7. Probability of correct classification as defined in (12). Cumulants were estimated on the sequence $\{z[n]\}$. The $F_{80 k, z}$ curve is the average of the values found in Figure 5.

that more data was needed in the case where features were obtained using $z[n]$. We calculate the probability of correct classification for each modulation at each $E_{s} / N_{0}$ as

$$
P_{c, \Omega_{i}}=\operatorname{Pr}\left(\operatorname{det} . \Omega_{i} \mid \Omega_{i}\right)
$$

\section{B. Signal Length}

A significant motivation in this study was to identify the viability of the classifiers in a real system. The cumulant can be better approximated with a longer sequence, but in a real system constraints such as energy, computation, and memory limitations provide an upper bound to the sequence length. Additionally, cumulants are affected by the total constellation rotation in the IQ plane during signal capture. Therefore, it is important to identify the minimum sequence length needed to achieve a certain level of performance. 


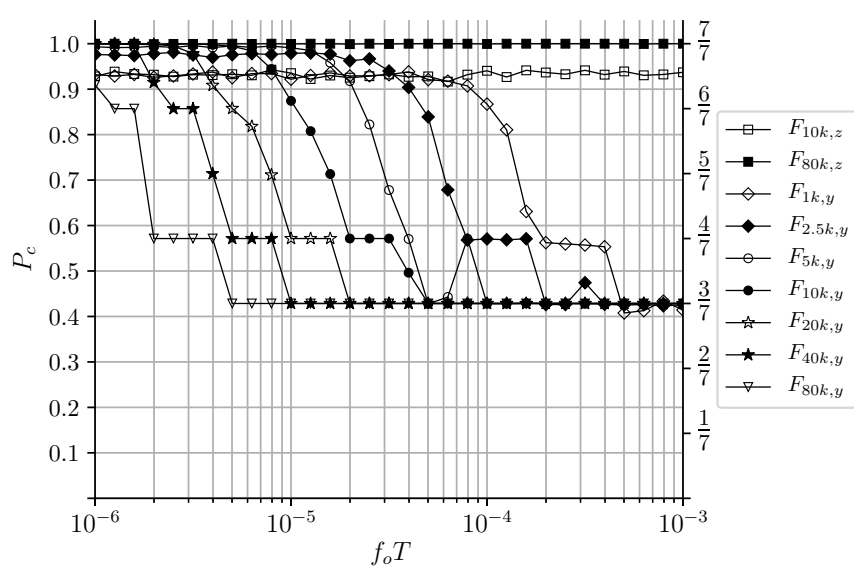

Fig. 8. Impact of a normalized, constant frequency offset. $E_{s} / N_{0}$ is fixed at $6 \mathrm{~dB}$.

Performance as a function of sequence length is evaluated by generating 1,000 instances of $F_{N, y}$ or $F_{N, z}$, per $E_{s} / N_{0}$ value, per modulation type, with zero frequency offset, a random constant phase offset and perfect knowledge of $E_{s} / N_{0}$. The probability of correct classification is calculated by

$$
P_{c}=\frac{\sum_{i} P_{c, \Omega_{i}}}{|\Omega|}
$$

Figure 6 and Figure 7 display the results for classifiers trained on $F_{N, y}$ and $F_{N, z}$ respectively. Classification based on $F_{N, z}$ required approximately $15-20$ times more data than $F_{N, y}$ for a similar level of performance. Given the motivations to capture short sequences, it is desirable to utilize $y[n]$ to estimate cumulant values; however, as will be shown in Section IV-C, cumulant estimations based on $y[n]$ are highly susceptible to frequency offset errors.

\section{Frequency Offset}

In an operational setting, we will have an unknown frequency offset. This frequency offset degrades the probability of classification when using the feature vector $F_{N, y}$. This occurs when the IQ constellation, on which the cumulants are operated, rotates during the capture period. The signal $y[n]$ will rotate during capture in the presence of a non-zero $f_{o}$. The signal $z[n]$ converts a fixed frequency offset to a fixed phase and, because we are using cumulant magnitudes, this constant phase does not impact results. However, $z[n]$ will experience rotation during capture in the presence of a timedependent frequency offset.

To evaluate the case of a fixed frequency offset, we generate a 31 point vector of frequency offsets in the range $\left[10^{-6}, 10^{-3}\right]$. Then, we generate 300 instances of $F_{N, y}$ and $F_{N, z}$ for each modulation, at $E_{s} / N_{0}=6 \mathrm{~dB}$, for each point in the frequency offset vector. The $E_{s} / N_{0}$ feature was set to $6 \mathrm{~dB}$ and a random fixed phase offset was applied. The results are shown in Figure 8, where we plot the probability of correct classification (12) against the normalized frequency offset.
As described earlier, the signal $z[n]$ converts a constant frequency offset to a fixed phase offset. In most real-world applications, there is often a time-dependent frequency offset and this time dependency will cause $z[n]$ to rotate in the IQ plane during signal capture. In previous S-band experiments [14] on the International Space Station (ISS), we observed a maximum $\frac{d f_{o}}{d t}=700 \frac{\mathrm{Hz}}{\mathrm{sec}}$. We used this maximum to approximate the phase accumulated during a capture due to a frequency offset by assuming a worst case scenario where this is constant over the capture period. Due to (4), a phase shift is applied equal to $2 \pi f_{o} T$. If $f_{o}=\alpha+\beta n T$, the time-dependent phase term is $2 \pi \beta n T^{2}$ and the total phase accumulation is $2 \pi \beta N T^{2}$. In the case where $\beta=700, N=80 k$, and $T=10^{-6}$, phase rotation for the sequence $\{z[n]\}$ is negligible.

\section{Es/No Estimation Error}

Up to this point, we have assumed perfect knowledge of $E_{s} / N_{0}$. In previous cumulant-based classifiers [3], [5], $r[n]$ is scaled by a value proportional to the signal-to-noise ratio (SNR) such that the received symbols have unit energy. Our method differs from this by normalizing $r[n]$ to unit energy and then including $E_{s} / N_{0}$ as a parameter in the feature vector. In either case, inaccuracies in SNR approximation will degrade classification performance and therefore warrants investigation.

To evaluate classifier performance as a function of $E_{s} / N_{0}$ approximation error, we add a $\Delta E_{s} / N_{0}$ to the true $E_{s} / N_{0}$ values in the feature vectors created for Section IV-A. The $\Delta E_{s} / N_{0}$ values are a 201 point, linearly sampled array in the range $[-5,5] \mathrm{dB}$. Early results showed that underestimation was more detrimental to classification ability than overestimation. Assuming the chosen estimator is symmetric about the true $E_{s} / N_{0}$, it makes sense to take the worst case for a given error magnitude. The results for $F_{5 k, y}$ and $F_{80 k, z}$ are presented in Figures 9 and 10, for signals with a true $E_{s} / N_{0}$ of $\{3,6,9,12,15\} \mathrm{dB}$.

These results show that classification is most sensitive to approximation error at low $E_{s} / N_{0}$ values. At $3 \mathrm{~dB}$ true $E_{s} / N_{0}$, both $F_{5 k, y}$ and $F_{80 k, z}$ dropped below $90 \%$ classification probability once $\left|\Delta E_{s} / N_{0}\right|$ was larger than $0.35 \mathrm{~dB}$. While at $20 \mathrm{~dB}$ true $E_{s} / N_{0}$, classification probability remains above $90 \%$ with errors approaching $3 \mathrm{~dB}$.

In an attempt to reduce our dependency on $E_{s} / N_{0}$ accuracy, we trained a classifier while adding noise to the $E_{s} / N_{0}$ feature. This method did reduce classification error due to $E_{s} / N_{0}$ approximation error, but at the expense of overall classification performance. We do not show these results here, but this may warrant further investigation if $E_{s} / N_{0}$ estimation becomes a limiting issue in a future system. We also leave the selection of a specific $E_{s} / N_{0}$ estimator as future work.

\section{E. Input Back-off}

During transmission, a signal is often driven beyond an amplifier's linear region. The optimal drive level is determined by weighing the benefit of increased amplifier output power 


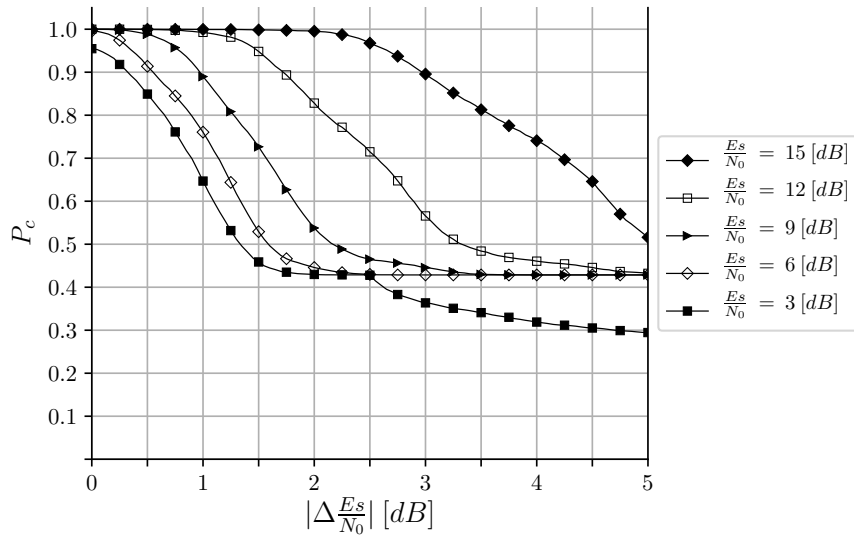

Fig. 9. Classification performance as a function of $E_{s} / N_{0}$ estimation error for $F_{5 k, y}$. For clarity, only every fifth evaluation point was marked by a plot marker.

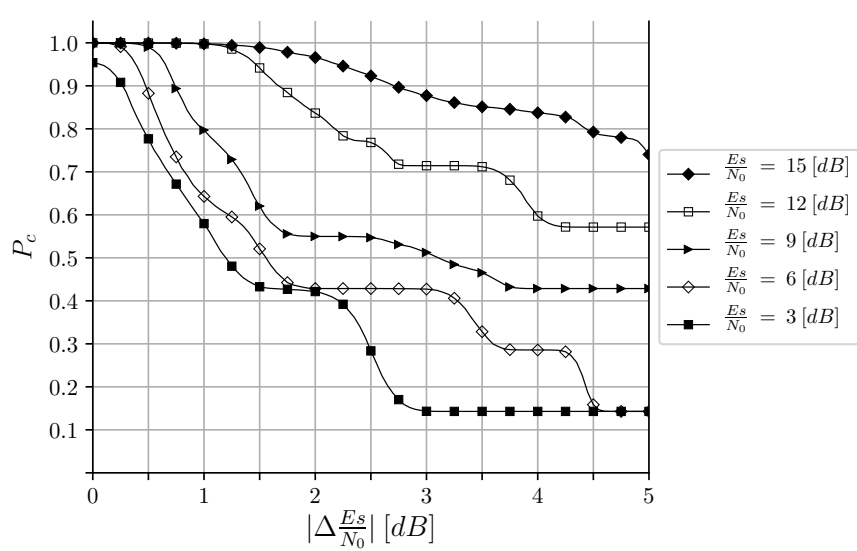

Fig. 10. Classification performance as a function of $E_{s} / N_{0}$ estimation error for $F_{80 k, z}$. For clarity, only every fifth evaluation point was marked by a plot marker.

against the error caused by modulation distortion. The distortion caused by operating the amplifier beyond the linear region is an amplitude-dependent compression and phase rotation in the IQ plane. To simulate amplifier effects, we used the Saleh model [15] with coefficients from an operational TWTA. In decibels, input back-off (IBO) is the difference between input power and the input power which maximizes output power. Symbols were selected from a power normalized alphabet and then scaled by $10^{-I B O / 20}$ before applying the Saleh model. After this, noise was added such that the signal had an $E_{s} / N_{0}$ equal to $6 \mathrm{~dB}$.

In Figure 11, we classify signals with a range of input backoffs, using the neural net trained on $F_{5 k, y}$. As expected, the PSK signals were not affected. This is because the PSK signals have a single amplitude level and the amplifier distortion is simply a phase rotation, after normalization. The APSK and QAM signals are greatly affected by amplifier distortion, with 64-QAM having a probability of correct classification which is less than $10 \%$ at $10 \mathrm{~dB}$ IBO. For reference, as IBO increases,

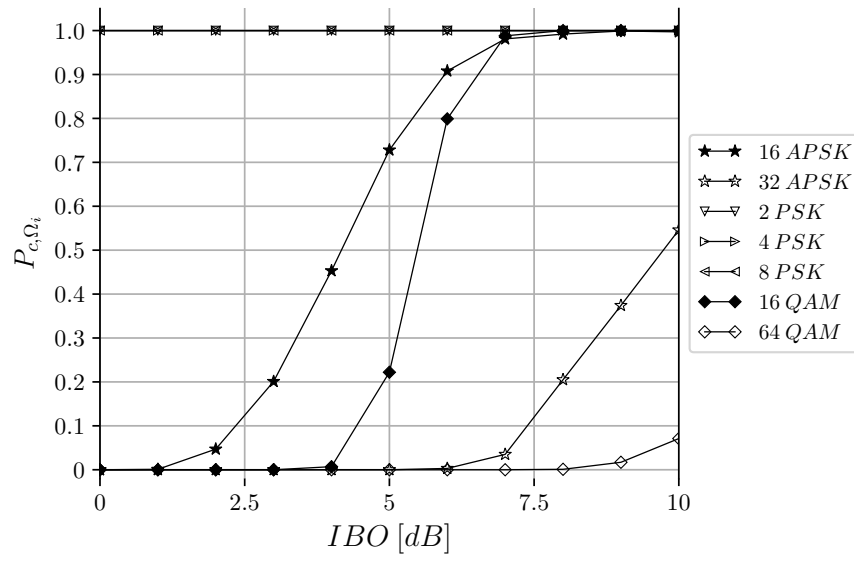

Fig. 11. Classification performance of $F_{5 k, y}$, as a function of input back-off when using a TWTA model.

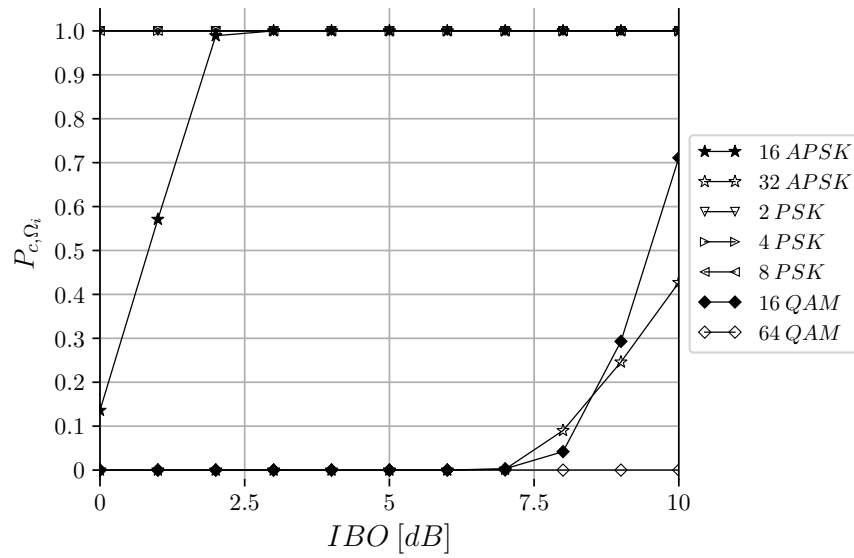

Fig. 12. Classification performance of $F_{80 k, z}$, as a function of input back-off when using a TWTA model.

we approach a slice in Figure 4 where $E_{s} / N_{0}=6 \mathrm{~dB}$. Similarly, we used the neural net trained on $F_{80 k, z}$ to classify signals distorted by our amplifier model, and those results are presented in Figure 12. In this case, as IBO increases we approach a slice in Figure 5.

These results show a dramatic degradation in performance. We expect that a solid-state amplifier would also reduce classification ability, but should be less pronounced due to solid state amplifiers having a more linear AM/AM curve and a flatter AM/PM curve. Regardless, this classifier requires the signal to experience a linear amplification process. Alternatively, if the user is able to parameterize the transmitting amplifier drive level, this feature could be used as input into the neural network. We expect that this would increase classification performance, given the drive level feature used is highly correlated with the transmitter's true drive level.

\section{F. DVB-S2 Physical Layer}

The DVB-S2 physical layer breaks the assumptions made on the symbol alphabet. In the description of $y[n]$ and $z[n]$, 


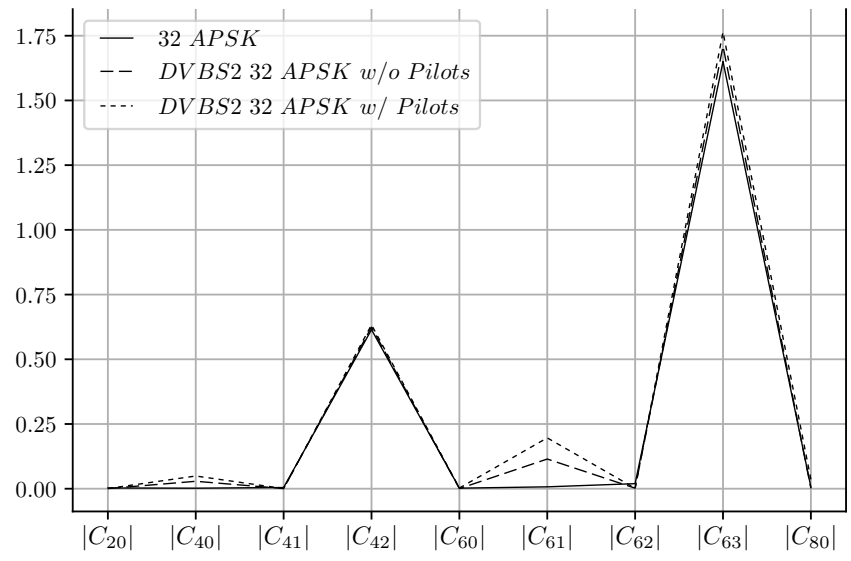

Fig. 13. Cumulant estimates of $y[n]$ for a $32-A P S K$ modulation, with ring ratios $\{2.72,4.87\}$, using a noiseless $1 \mathrm{M}$ length sequence. DVB-S2 signals are MODCOD 25.

we define the symbols $x[n]$ to belong to a single modulation alphabet, but DVB-S2 signals will not follow this definition. The DVB-S2 standard uses a MODCOD parameter, which describes the data frame's modulation and Forward Error Correction (FEC) code rate. The DVB-S2 header uses a $\frac{\pi}{2}$ 2-PSK modulation format and is appended to the beginning of each frame. The user may also enable pilot tones. These use a 4-PSK modulation scheme and are inserted into specific locations in the data frame [13]. This means that the sequence $\{x[n]\}$ will pull symbols from up to three alphabets. We continue to use $y[n]$ and $z[n]$ to describe the presence of the differentiating operation defined in (4).

The impact on cumulant estimates for a noiseless, zero frequency offset, 32-APSK modulation with ring ratios $\{2.72,4.87\}$, are displayed in Figures 13 and 14 for $y[n]$ and $z[n]$, respectively. For $y[n]$, the set of possible symbol locations is the concatenation of the data, header and pilot symbol alphabets. The signal $z[n]$ is more complicated as the possible symbol locations include the product of all possible combinations of symbols from the three sets.

To evaluate the impact of the DVB-S2 physical layer on classification ability, we generated 1,000 sequences of length 5,000 using the $y[n]$ model at $E_{s} / N_{0}$ values of $\{6,10,15,20\}$ $\mathrm{dB}$, for MODCODs $\{1,12,18,19,20,21,22,24,25,26,27\}$, for cases with pilots enabled and disabled. Classification was done using $F_{5 k, y}$ and the results were compared to signals where $x[n]$ belonged solely to the modulation alphabet defined by the MODCOD. Similarly, we repeated this for the $z[n]$ case using sequence lengths of 80,000 and classified on $F_{80 k, z}$.

Over the sample points described, $y[n]$ signals with pilots enabled and disabled maintained classification probabilities equal to or higher than $88 \%$ and $92 \%$, respectively. The largest change in classification probability for $y[n]$ signals was $-5.3 \%$ at $E_{s} / N_{0}$ equal to $6 \mathrm{~dB}$ for MODCOD 27 with pilots disabled, and $-8.8 \%$ at $E_{s} / N_{0}$ equal to $6 \mathrm{~dB}$ for MODCOD 27 with pilots enabled.

For $z[n]$ signals, the largest change in classification prob-

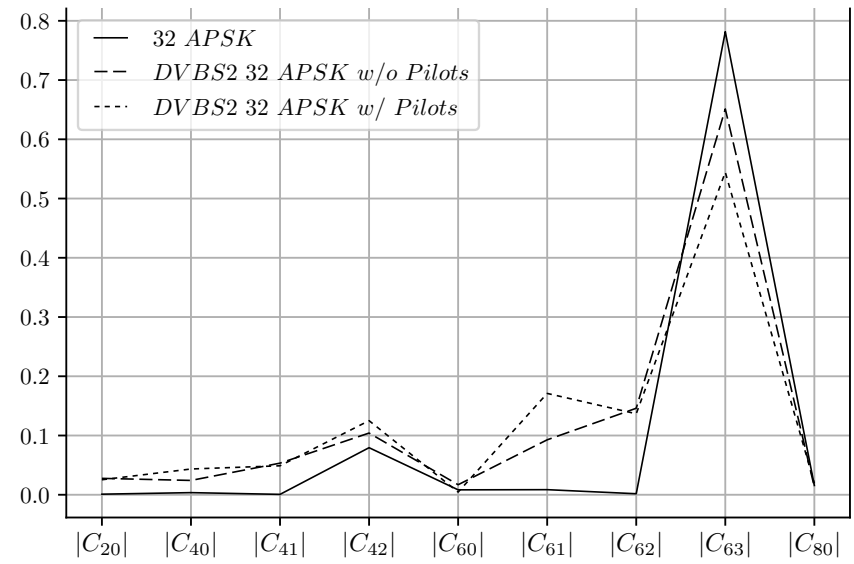

Fig. 14. Cumulant estimates of $z[n]$ for a $32-A P S K$ modulation, with ring ratios $\{2.72,4.87\}$, using a noiseless $1 \mathrm{M}$ length sequence. DVB-S2 signals are MODCOD 25.

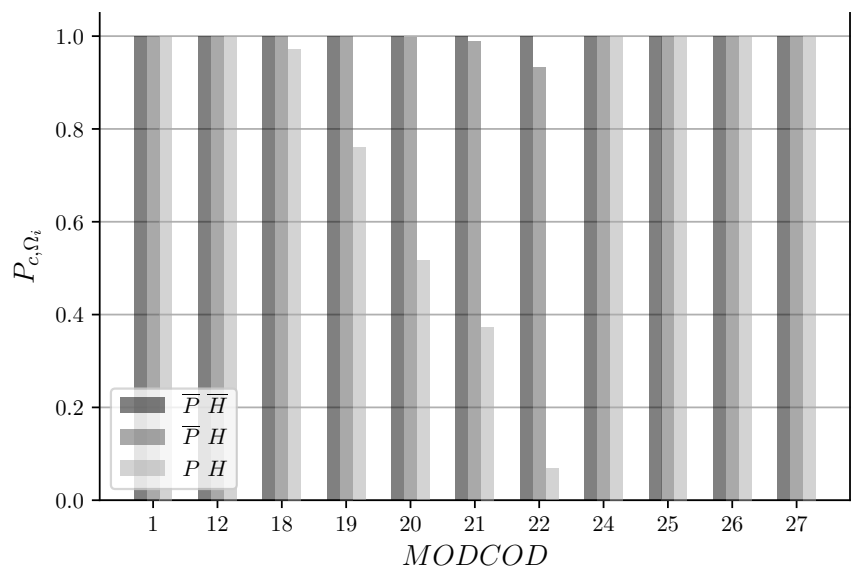

Fig. 15. Probability of classification using $F_{80 k, z}$ at $E_{s} / N_{0}$ equal to 20 $\mathrm{dB}$. Pilot tones and header sequences are represented by $\mathrm{P}$ and $\mathrm{H}$, with the overbar representing that element being not present in the sequence.

ability occurred at $E_{s} / N_{0}$ equal to $20 \mathrm{~dB}$ and is displayed in Figure 15. For the $E_{s} / N_{0}$ set $\{6,10,15\} \mathrm{dB}$, with pilots disabled, classification probability degraded by less than $2 \%$ for all MODCODs. With pilots enabled, the largest change in classification probability was $-6.7 \%$ at $E_{s} / N_{0}$ equal to $15 \mathrm{~dB}$ for MODCOD 22.

\section{CONCLUSION}

We have highlighted several areas that will need consideration before this type of automatic classification scheme can be implemented in a real system. Engineers will need to identify the minimum sequence length needed to obtain the desired level of performance, and then use that information to determine the accuracy needed during carrier removal. If carrier removal cannot be performed to a high enough degree of precision, $z[n]$ can be used as an alternative, but at the expense of longer captures. We have also provided an analysis of classification performance as a function of $E_{s} / N_{0}$ 
approximation error, which sets bounds on the required performance of an $E_{s} / N_{0}$ estimator. This classification scheme is not resilient to nonlinear amplification, due to the modulation set including modulations with multiple amplitude levels. The DVB-S2 physical layer will impact cumulant values and therefore degrades classification ability. We found this to be most detrimental in high SNR environments when using the $z[n]$ signal type.

\section{REFERENCES}

[1] O. Dobre, A. Abdi, Y. Bar-Ness and W. Su, "Survey of automatic modulation classification techniques: classical approaches and new trends," IET Communications, vol. 1, no. 2, pp. 137-156, 2007.

[2] A. Nandi and E. Azzouz, "Modulation recognition using artificial neural networks," Signal Processing, vol. 56, no. 2, pp. 165-175, 1997.

[3] A. Swami and B. Sadler, "Hierarchical digital modulation classification using cumulants," IEEE Transactions on Communications, vol. 48, no. 3 , pp. $416-429,2000$.

[4] M. Wong and A. Nandi, "Automatic digital modulation recognition using network and genetic algorithm," Signal Processing, vol. 84, no. 2, pp. 351-365, 2004.

[5] M. Flohberger, W. Gappmair and O. Koudelka, "Modulation classifier for signals used in satellite communications," in 5th Advanced Satellite Multimedia Systems Conference and the 11th Signal Processing for Space Communications Workshop, 2010.

[6] C.M. Spooner, "Classification of co-channel communication signals using cyclic cumulants," Proc. Asilomar, pp. 531-536, 1996.

[7] —_, "Automatic radio-frequency environment analysis," in Conf. Record of the Thirty-Fourth Asilmar Conf., 2000.

[8] — " "On the utility of sixth-order cyclic cumulants for rf signal classification," Proc. Asilomar, pp. 890-897, 2001.

[9] O.A. Dobre, Y. Bar-Ness, and S. Wei, "Higher-Order Cyclic Cumulants for High Order Modulation Classification," in Military Communications Conference, Boston, MA, 2003.

[10] E. Rebeiz, Y. Fang-Li, P. Urriza, D. Markovic, and D. Cabric, "Energyefficient processor for blind signal classification in cognitive radio networks," IEEE Transactions on Circuits and Systems-I: Regular Papers, vol. 61, no. 2, pp. 587-599, 2014.

[11] A. Fehske, J. Gaeddert, and J. Reed, "A new approach to signal classification using spectral correlation and neural networks," in New Frontiers in Dynamic Spectrum Access Networks, 2005. DySPAN 2005. 2005 First IEEE International Symposium, 2005, pp. 144-150.

[12] S. Sobolewski, W. Adams and R. Sankar, "Universal Nonhierarchical Automatic Modulation Recognition Techniques for Distinguishing Bandpass Modulated Waveforms Based on Signal Statistics, Cumulant, Cyclostationary, Multifractal and Fourier-Wavelet Transforms Features," in IEEE Military Communications Conf., 2014.

[13] Digital Video Broadcasting (DVB); Second generation framing structure, channel coding and modulation systems for Broadcasting, Interactive Services, News Gathering and other broadband satellite applications; Part1: (DVB-S2), Std. ETSI EN 302 307-1 V1.4.1 (2014-2011).

[14] J.A. Downey, D. Mortensen, M. Evans, J. Briones, and N. Tollis, "Adaptive Coding and Modulation Experiment with NASA's Space Communication and Navigation Testbed," in 34th AIAA International Communications Satellite Systems Conference, 2016.

[15] A.A.M. Saleh, "Frequency-independent and frequency-dependent nonlinear models of twt amplifiers," IEEE Transactions on Communications, pp. $1715-1720,1981$.

[16] J.A. Downey, J.M. Downey, R. Reinhart, M. Evans, and D. Mortensen, "Bandwidth-Efficient Communication through $225 \mathrm{MHz}$ Ka-band Relay Satellite Channel," in 34th AIAA International Communications Satellite Systems Conference, 2016.

[17] C. Long, K. Chugg, and A. Polydoros, "Further results in maximum likelihood classification of QAM signals," in Proc. MILCOM, Long Branch, NJ, 1994, pp. 57-61.

[18] J. Mendel, "Tutorial on higher-order statistics (spectra) in signal processing and system theory: Theoretical results and some applications," Proceedings of the IEEE, vol. 79, no. 3, pp. 278-305, 1991.

[19] D. P. Kingma and J. Ba, "Adam: A method for stochastic optimization," CoRR, vol. abs/1412.6980, 2014. [Online]. Available: http://arxiv.org/abs/1412.6980 